\title{
Fishes of Paranaguá Estuarine Complex, South West Atlantic
}

\author{
Ana Carolina dos Passos ${ }^{1,8}$, Riguel Feltrin Contente ${ }^{2}$, Ciro Colodetti Vilar de Araujo ${ }^{3}$, \\ Felippe Alexandre Lisboa de Miranda Daros, Henry Louis Spach, \\ Vinícius Abilhôa ${ }^{6}$ \& Luis Fernando Fávaro ${ }^{7}$
}

\author{
${ }^{1}$ Laboratório de Biologia de Peixes, Programa de Pós-graduação em Sistemas Costeiros e Oceânicos, \\ Departamento de Ciências da Terra, Centro de Estudos do Mar, Universidade Federal do Paraná - UFPR, \\ CP 50002, CEP 83255-000, Pontal do Paraná, PR, Brasil \\ ${ }^{2}$ Laboratório de Ecologia Reprodutiva, Instituto Oceanográfico, Universidade de São Paulo - USP, \\ CEP 05508-120, São Paulo, SP, Brasil \\ ${ }^{3}$ Programa de Pós-graduação em Ecologia e Conservação, Universidade Federal do Paraná - UFPR, \\ CP 19031, CEP 81531-980, Curitiba, PR, Brasil \\ ${ }^{4}$ Laboratório de Biologia de Peixes, Programa de Pós-graduação em Zoologia, Departamento de Ciências \\ da Terra, Centro de Estudos do Mar, Universidade Federal do Paraná - UFPR, CP 50002, \\ CEP 83255-000, Pontal do Paraná, PR, Brasil \\ ${ }_{5}^{5}$ Laboratório de Biologia de Peixes, Departamento de Ciências da Terra, Centro de Estudos do Mar, \\ Universidade Federal do Paraná - UFPR, CP 50002, CEP 83255-000, Pontal do Paraná, PR, Brasil \\ ${ }^{6}$ Laboratório de Ictiologia, Museu de História Natural Capão da Imbuia, Secretaria Municipal do Meio \\ Ambiente, Prefeitura Municipal de Curitiba, CEP 82810080, Curitiba, PR, Brasil \\ ${ }^{7}$ Laboratório de Reprodução e Comunidade de Peixes, Universidade Federal do Paraná - UFPR, \\ CP 19031, CEP 81531-980, Curitiba, PR, Brasil \\ ${ }^{8}$ Corresponding author: Ana Carolina dos Passos, e-mail: anacarolpassos@yahoo.com.br
}

PASSOS, A.C., CONTENTE, R.F., VILAR, C.C., DAROS, F.A., SPACH, H.L., ABILHÔA, V. \& FÁVARO, L.F. Fishes of Paranaguá Estuarine Complex, South West Atlantic. Biota Neotrop. 12(3): http://www. biotaneotropica.org.br/v12n3/en/abstract?inventory+bn01312032012

\begin{abstract}
The objective of this work was to present an updated checklist of the currently known fishes in the Paranaguá Estuarine Complex (PEC) and provides comments on conservation status for the treated species. We used a large dataset derived from a pool of studies which have been conducted within there along the last 30 years. Each study was based on monthly samplings and conducted in several estuarine habitat; thus, the pool covers practically all estuarine habitats and takes into account the seasonal cycle in the system. The PEC ichthyofauna represents a mixture between that fauna typical from the tropical Brazilian coast and that with affinities of temperate Argentinean and Uruguayan zones. The PEC harbors a rich fish fauna of 213 species, inserted in the families that are common along the Brazilian coast. Only a minor part (8\%) of the PEC fish fauna was evaluated as regards the conservation status, mostly because of the lack of basic biological and ecological information for most species. Despite part of the among-estuaries differences are due to different and incomplete sampling efforts, the richness in the PEC is surprisingly higher than other systems in Brazil and around world, which emphasize the importance of the region for global biodiversity conservation.
\end{abstract}

Keywords: ichthyofauna, species list, Paraná, West Atlantic.

PASSOS, A.C., CONTENTE, R.F., VILAR, C.C., DAROS, F.A., SPACH, H.L., ABILHÔA, V. \& FÁVARO, L.F. Peixes do Complexo Estuarino de Paranaguá, Atlântico Sul Oeste. Biota Neotrop. 12(3): http://www. biotaneotropica.org.br/v12n3/pt/abstract?inventory+bn01312032012

Resumo: O objetivo deste trabalho é apresentar uma lista de espécies atualizada a partir do conhecimento atual dos peixes no Complexo Estuarino de Paranaguá (CEP), com comentários sobre o status de conservação das espécies. Nós utilizamos um grande conjunto de dados derivados de diversos estudos conduzidos na região ao longo dos últimos 30 anos. Cada estudo foi baseado em amostragens mensais realizadas em diferentes habitats estuarinos. Dessa forma, os dados abrangem praticamente todos os habitats estuarinos e leva em conta o ciclo sazonal no sistema. A ictiofauna do CEP representa uma mistura entre a fauna típica da costa tropical brasileira e da fauna com afinidade com as zonas temperadas argentinas e uruguaias. O CEP abriga 213 espécies, inseridas em famílias que são comuns ao longo da costa brasileira. Apenas uma pequena parte ( $8 \%$ ) das espécies foi avaliada quanto ao status de conservação, principalmente por causa da falta de informações biológicas e ecológicas básicas para a maioria das espécies. Apesar de parte das diferenças entre estuários ser devido a diferentes e incompletos esforços amostrais, a riqueza do CEP é surpreendentemente elevada em comparação com outros sistemas do Brasil e ao redor do mundo, o que enfatiza a importância da região para a conservação da biodiversidade global. Palavras-chave: ictiofauna, lista de espécies, Paraná, Atlântico Oeste. 


\section{Introduction}

The Paranaguá Estuarine Complex (PEC), situated on the coast of Paraná state (Brazil) $\left(25^{\circ} 16^{\prime}\right.$ and $25^{\circ} 34^{\prime} \mathrm{S}$ and $48^{\circ} 17^{\prime}$ and $\left.48^{\circ} 42^{\prime} \mathrm{W}\right)$, represents the southern sector of one of the last and least impacted, large Brazilian coastal ecosystems, the subtropical Iguape-Cananéia-Paranaguá estuarine system. This system harbors an important biodiversity, as it is inserted in a global biodiversity hotspot, the southern sector of Brazil's Atlantic Forest Biosphere Reserve (Diegues 1995), and an abundance of socio-economically important fishery stocks (Lana et al. 2001). Several surveys and ecological studies on the fish fauna, most of which based on a monthly sampling design including a diversity of estuarine habitats, have been conducted in the PEC along the last 30 years (e.g. Corrêa et al. 1986, Spach et al. 2003, Félix et al. 2007, Queiroz et al. 2007, Schwarz Junior. et al. 2007, Barletta et al. 2008, Contente et al. 2011). Sampling several areas within several temporal scales increases the species detectability and, thus, our ability to estimate the species richness of a given system (Magurran 2003); thus, such robust spatio-temporal information included in the data derived from the pool of these studies provide a unique opportunity to produce a full list of fish species for this system. A compilation of this nature has twofold: (I) helping to improve our understanding on the geographical distribution and macro-ecological traits of the SW Atlantic estuarine fishes (Barletta \& Blaber 2007); and (II) supporting conservation efforts (Barletta et al. 2010).
Particular concern must be placed to the region's fish biodiversity that is faced to serious treats, notably due to overfishing, introduction of non-native species, and habitat loss (Lana et al. 2001, Vitule et al. 2006, Caires et al. 2007), and a full check-list of species may be an important tool in impact assessments. For instance, dredgings and buildings of ports result in large impact and an ecosystem scale check-list may serve as a parameter against which the potentially affected pattern of the fish fauna may be compared, thus helping to assess the strength of impact (Sheaves 2006, Barletta et al. 2010). The objective of the present work is, therefore, to present an updated checklist of the currently known fishes in the PEC. Additionally, we provided comments on conservation status for the treated species.

\section{Materials and Methods}

\section{Study area}

The PEC (Figure 1) has an area of $612 \mathrm{~km}^{2}$ (see map in Lana et al. 2001) characterized by distinct areas and densities of drainage, tidal flats and mean depths (Noernberg et al. 2004). The system has a diversity of habitats, like tidal flats, channels, mangroves (mainly composed by Rhizophora mangle, Avicennia schaueriana, Laguncularia racemosa, and Conocarpus erectus; Lana et al. 2001) fringed by Spartina alterniflora bank salt-marshes, tidal creeks, estuarine beaches, rivers, and rock shores near the mouth of the

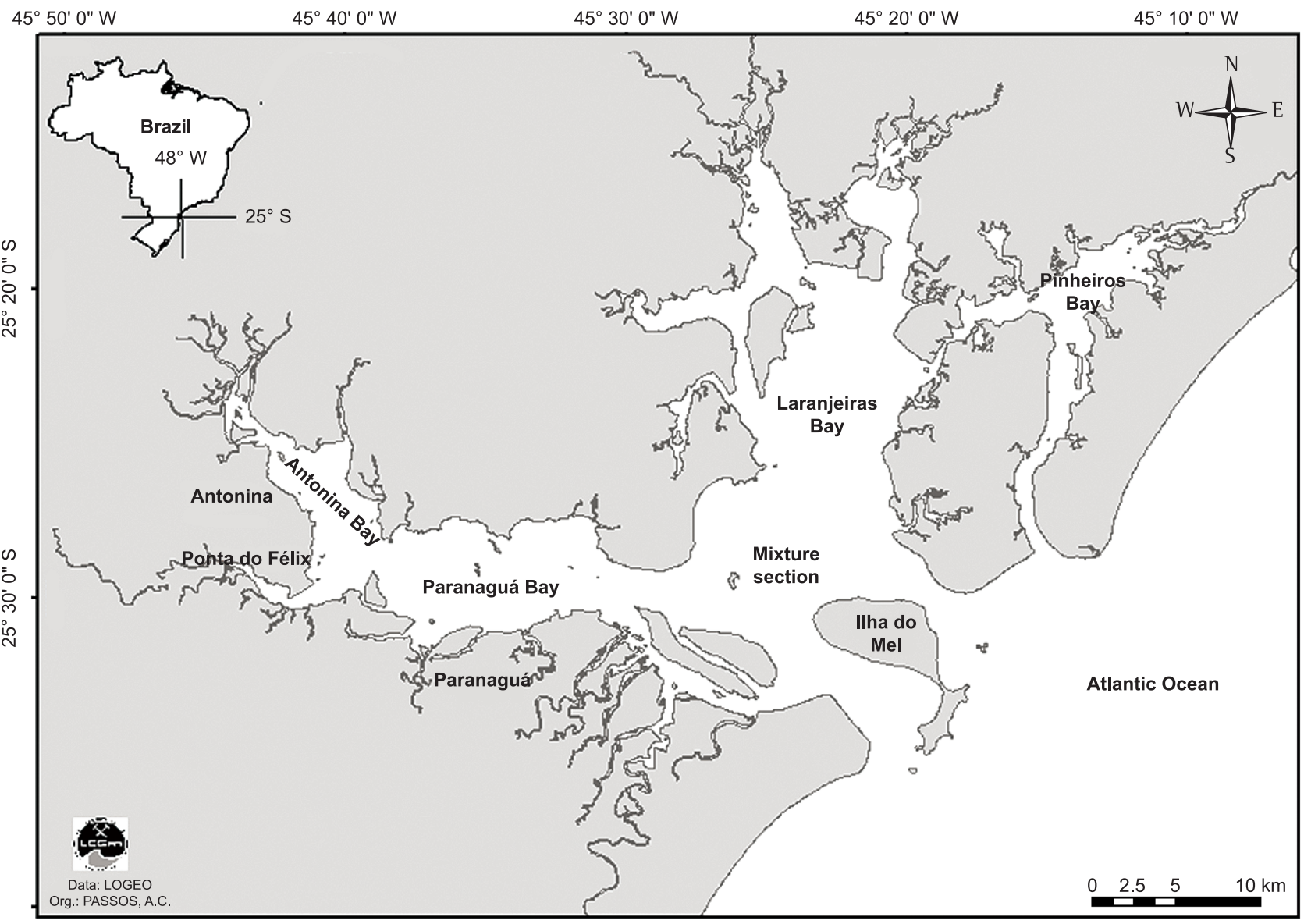

Figure 1. Map of Paranaguá Estuarine Complex, showing the five central sectors (Antonina, Paranaguá, Laranjeiras, mixing zone and Pinheiros); and the position of port areas (ports of Paranaguá, Antonina and Ponta do Félix).

Figura 1. Mapa do Complexo Estuarino de Paranaguá, mostrando as Baías de Antonina, Paranaguá, das Laranjeiras, Pinheiros e setor de mistura; e a localização dos Portos de Paranaguá, Antonina e Ponta do Félix. 
estuary. The PEC, a partially mixed estuary with semidiurnal tides and diurnal inequality (Knoppers et al. 1987), is connected to the Cananéia Estuarine Complex, in the north, by the Ararapira Channel and to the Atlantic Ocean, in the east, by Sueste Channel and Galheta Channel. The climate of the region is tropical (transition), with a mean annual rainfall of $2500 \mathrm{~mm}$ (maximum $3500 \mathrm{~mm}$ ). The rainy season typically starts at the end of spring and lasts until nearly the end of summer. The dry season lasts from the end of autumn to the end of winter, but is interrupted by a short low-intensity rainy period that occurs at the beginning of winter (Lana et al. 2001). To further details on the system, see Lana et al. (2001) and Marone et al. (2005).

\section{Data collection and treatment of the data}

This work is based on the compilation of data from unpublished $\mathrm{PhD}$ thesis and master dissertations (i.e. Abilhôa 1998, Pinheiro 1999, Fávaro 2004, Nakayama 2004, Stefanoni 2007, Pichler 2009) and from the literature dealing with the PEC fish community (e.g. Corrêa et al. 1986, Godefroid et al. 1997, 1999, Santos et al. 2002, Vendel et al. 2002, Spach et al. 2003, Vendel et al. 2003, Spach et al. 2004a, b, 2006, Félix et al. 2007, Queiroz et al. 2007, Schwarz Junior et al. 2007, Barletta et al. 2008, Oliveira Neto et al. 2008, Pichler et al. 2008, Cortellete et al. 2009, Hackradt et al. 2009, Ignácio \& Spach 2009, Contente et al. 2011). It is important to highlight that this study is representative of most habitats (e.g. tidal flats, channels, vegetated wetlands, tidal creeks, estuarine beaches, rivers) and the extension of the system, i.e., from the representative, upper tidal freshwater reaches (like upper Antonina Bay Estuary and upper Guaragaçu River Estuary) to the mouths of the estuary (like Pontal do Sul and Ilha das Peças beaches). Practically all studies were took place with monthly samplings along the seasonal cycle. The species in this study were reviewed as regards the taxonomic classification and the nomenclature based on Marceniuk (2005), Craig \& Hastings (2007), Smith \& Craig (2007), Eschmeyer (2010), Carvalho-Filho et al. (2010), Figueiredo et al. (2010), and Menezes et al. (2010). Mugil sp. was used for the species usually identified under the invalid name Mugil gaimardianus (Menezes et al. 2003). The orders and families were listed in phylogenetic order according to Eschmeyer (2010) and the species were organized within each family in alphabetical order. To analyze zoogeographic affinities, the distribution of each species was verified from the literature and then inserted into the adapted categories based on Floeter et al. (2008) and Luiz Junior et al. (2008) as follows: $\mathrm{CT}=$ Circumtropical, $\mathrm{TA}=$ Trans-Atlantic (western and eastern Atlantic Ocean), WA = Western Atlantic (northern and southern Atlantic Ocean), SWA = Southern West Atlantic (from northern Brazil to Argentina), SSWA = Southern South West Atlantic (species with temperate affinities occurring from Argentina and Uruguay to the south and southeast of Brazil), $\mathrm{Ca}=$ Caribbean (from Florida state to Venezuela), $\mathrm{Br}=$ Brazilian Province (area between the Orinoco Delta in Venezuela and Santa Catarina in Brazil), EA = Eastern Atlantic and EP = Eastern Pacific. The status of species conservation was based both on the International Union for Conservation of Nature list (International... 2012) and the Ministry of the Environment list for endangered fauna (Brasil 2004, 2008).

\section{Results and Discussion}

The ichthyofauna of the PEC consists of 213 species, distributed in 21 orders and 65 families (Table 1). A total of $97 \%$ (208 spp.) are Actinopterygii and 3\% (5), Elasmobranchii. Twenty species (i.e. H. robertii, O. vespertilio, A. clupeoides, A. januaria, G. ocellatus, S. brasiliensis, S. plumieri, A. brasilianus, M. bonaci, L. synagris, A. probatocephalus, C. penna, P. cromis, U. parvus, G. brasiliensis, E. pisonis, S. picudilla, A. solandri, P. patagonicus and T. microphthalmus) were recorded for the first time for the PEC. Perciformes (116) dominated in number of species, followed by Clupeiformes (20), Pleuronectiformes (17), and Syngnathiformes (8). The most speciose family was Sciaenidae (23), followed by Carangidae (17), Engraulidae (12), Gobiidae (9), Haemulidae (9), and Paralichthyidae (9). This is supported by Andrade-Tubino et al. (2008) that state such families among the most important in Brazilian coast, and by Vieira \& Musick (1994), which reveal them as the most conspicuous in SW Atlantic estuarine fish assemblages. Carangidae and Sciaenidae were also the two most speciose families in the two large estuaries near the PEC, the Guaratuba Bay, located just south of the PEC, and the Babitonga Bay, located $70 \mathrm{Km}$ south of the PEC (Chaves \& Corrêa 1998, Chaves \& Vendel 2001, Vilar et al. 2011). Anchoa and Cynoscion had six species each and were the richest genera in the PEC. The following were Mugil, with five species, and Ctenogobius, Paralichthys, Sphoeroides, Sphyraena and Trachinotus, with four species each. This pattern was not found in such nearby estuaries, as Ctenogobius, followed by Eucinostomus and Oligoplites were the richest genera in Guaratuba (Chaves \& Corrêa 1998, Chaves \& Vendel 2001), and Mycteroperca, Mugil, Anchoa, Cynoscion, Eucinostomus and Sphoeroides were those most rich in Babitonga (Vilar et al. 2011). Comparing the species composition of PEC with that of Babitonga and Guaratuba estuaries reveals a relatively low number shared (just 35\%) and relatively a high number of exclusive species (about 40\%) of the PEC. This is unexpected because the distribution for most species occurring in all such estuaries overlaps. Such taxonomic differences are most likely due to differences in number of fish survey as well as in fish sampling gear and strategies (see discussion below).

In terms of number of species, the total species richness in the PEC is higher than those reported for large estuaries in Western Atlantic [Guaratuba Bay, southern Brazil (87 spp.; Chaves \& Corrêa 1998, Chaves \& Vendel 2001); Río de la Plata estuary, northern Argentina (60 spp.; Jaureguizar et al. 2004); Caeté River estuary, northern Brazil (82spp.; Barletta et al. 2005), Sergipe River estuary (136 spp.; Alcântara 2006), Curuçá estuary (98 spp.; Hercos 2006, Giarrizzo \& Krumme 2007, Sarpedonti et al. 2008), Babitonga Bay (152 spp.; Vilar et al. 2011), Estuary of Mataripe area (36 spp.; Dias et al. 2011) and Paraguaçu River estuary (124 spp.; Reis-Filho et al. 2010)] as well as for other large, permanently open, tropical estuaries around world (number of species ranging from 81 to 197, Blaber 2002), including those of estuaries from the species-rich Indo Pacific biogeographical zones. Moreover, the PEC has a comparable fauna to large coastal ecosystems, like Gulf of Carpentaria (237 spp.) (Blaber et al. 1990) and Embley estuary (203 spp.) (Barletta \& Blaber 2007), which has a large diversity of estuarine habitats, similar to that of PEC. These differences in richness and taxonomic composition may be difficult to explain. Multiple area specific synergic factors act in determining fish fauna patterns, including diversity of estuarine habits, rainfall pattern, hydrograph, oceanographic patterns, and historic dispersion pattern of taxa and so on. These operate in different intensity and scale, producing very distinct fish fauna patterns. For example, in a continental scale, the estuarine area may be critical to determine the fish richness in accordance with the species-area theory. This theory states that, the larger a given system, the larger the number of species, because the number of habitat tend to increase with the area. In fact, the PEC is larger $\left(612 \mathrm{~km}^{2}\right)$ than the Guaratuba Bay $\left(48 \mathrm{~km}^{2}\right)$, Babitonga Bay $\left(130 \mathrm{~km}^{2}\right)$, Sergipe River estuary $\left(47.1 \mathrm{~km}^{2}\right)$ and Paraguaçu River estuary $\left(127.9 \mathrm{~km}^{2}\right)$ and this may explain, at least in part, the higher richness in PEC. However, a considerable part of such differences among tropical fish faunas can be attributed to the incomplete effort of surveys on all range of estuarine habitats 
Table 1. Taxonomic classification of the ichthyofauna recorded in the Paranaguá Estuarine Complex, Southwest Atlantic.

Tabela 1. Classificação taxonômica da ictiofauna encontrada no Complexo Estuarino de Paranaguá, Atlântico Sul Oeste.

\begin{tabular}{|c|c|c|}
\hline Orders/Families/Species & Geographic distribution & Source \\
\hline \multicolumn{3}{|l|}{ Torpediniformes } \\
\hline \multicolumn{3}{|l|}{ Narcinidae } \\
\hline Narcine brasiliensis (Olfers 1831) & WA & Spach et al. (2004a) \\
\hline \multicolumn{3}{|l|}{ Rajiformes } \\
\hline \multicolumn{3}{|l|}{ Rhinobatidae } \\
\hline Rhinobatos horkelii Müller \& Henle $1841 \dagger$ & SWA & Barletta et al. (2008) \\
\hline R. percellens (Walbaum 1792) & TA & Pichler et al. (2008) \\
\hline \multicolumn{3}{|l|}{ Dasyatidae } \\
\hline Dasyatis guttata (Bloch \& Schneider 1801) & $\mathrm{Ca}+\mathrm{Br}$ & Schwarz Junior et al. (2007) \\
\hline \multicolumn{3}{|l|}{ Gymnuridae } \\
\hline Gymnura altavela (Linnaeus 1758) & TA & Schwarz Junior et al. (2007) \\
\hline \multicolumn{3}{|l|}{ Elopiformes } \\
\hline \multicolumn{3}{|l|}{ Elopidae } \\
\hline Elops saurus Linnaeus 1766 & WA & Pichler et al. (2008) \\
\hline \multicolumn{3}{|l|}{ Albuliformes } \\
\hline \multicolumn{3}{|l|}{ Albulidae } \\
\hline Albula vulpes (Linnaeus 1758) & WA & Pichler et al. (2008) \\
\hline \multicolumn{3}{|l|}{ Anguilliformes } \\
\hline \multicolumn{3}{|l|}{ Muraenidae } \\
\hline Gymnothorax ocellatus Agassiz 1831 & $\mathrm{Ca}+\mathrm{SWA}$ & Nakayama (2004) \\
\hline \multicolumn{3}{|l|}{ Ophichthidae } \\
\hline Echiophis intertinctus (Richardson 1848) & WA & Spach et al. (2004a) \\
\hline Myrophis punctatus Lütken 1852 & WA & Spach et al. (2004a) \\
\hline Ophichthus gomesii (Castelnau 1855) & WA & Oliveira Neto et al. (2008) \\
\hline \multicolumn{3}{|l|}{ Congridae } \\
\hline Conger orbignianus Valenciennes 1837 & SSWA & Spach et al. (2004a) \\
\hline \multicolumn{3}{|l|}{ Muraenesocidae } \\
\hline Cynoponticus savanna (Bancroft 1831) & $\mathrm{Ca}+\mathrm{Br}$ & Spach et al. (2004a) \\
\hline \multicolumn{3}{|l|}{ Nettastomatidae } \\
\hline Hoplunnis tenuis Ginsburg 1951 & WA & Spach et al. (2004a) \\
\hline \multicolumn{3}{|l|}{ Clupeiformes } \\
\hline \multicolumn{3}{|l|}{ Clupeidae } \\
\hline Brevoortia sp. & $?$ & Godefroid et al. (1999) \\
\hline Chirocentrodon bleekerianus (Poey 1867) & $\mathrm{Ca}+\mathrm{Br}$ & Oliveira Neto et al. (2008) \\
\hline Harengula clupeola (Cuvier 1829) & WA & Pichler et al. (2008) \\
\hline H. jaguana Poey 1865 & WA & Godefroid et al. (1997) \\
\hline Opisthonema oglinum (Lesueur 1818) & WA & Pichler et al. (2008) \\
\hline Platanichthys platana (Regan 1917) & SSWA & Pichler et al. (2008) \\
\hline Sardinella brasiliensis (Steindachner 1879) $\dagger \dagger$ & SSWA & Pichler et al. (2008) \\
\hline \multicolumn{3}{|l|}{ Engraulidae } \\
\hline Anchoa filifera (Fowler 1915) & $\mathrm{Ca}+\mathrm{Br}$ & Godefroid et al. (1997) \\
\hline A. hepsetus (Linnaeus 1758) & WA & Barletta et al. (2008) \\
\hline A. januaria (Steindachner 1879) & $\mathrm{Br}$ & Pichler (2009) \\
\hline A. lyolepis (Evermann \& Marsh 1900) & WA & Pichler et al. (2008) \\
\hline A. spinifer (Valenciennes 1848) & $\mathrm{Ca}+\mathrm{Br}+\mathrm{EP}$ & Barletta et al. (2008) \\
\hline A. tricolor (Spix \& Agassiz 1829) & SWA & Pichler et al. (2008) \\
\hline Anchovia clupeoides (Swainson 1839) & $\mathrm{Ca}+\mathrm{Br}$ & Nakayama (2004) \\
\hline Anchoviella brevirostris (Günther 1868) & $\mathrm{Br}$ & Barletta et al. (2008) \\
\hline
\end{tabular}

Geographic distribution: $\mathrm{CT}=$ circuntropical, $\mathrm{TA}=$ Trans-Atlantic, WA $=$ Western Atlantic, $\mathrm{SWA}=$ Southern West Atlantic, $\mathrm{SSWA}=$ Southern South West Atlantic, $\mathrm{Ca}=$ Caribbean, $\mathrm{Br}=$ Brazilian Province, $\mathrm{EA}=$ Eastern Atlantic, $\mathrm{EP}=$ Eastern Pacific and $?=$ not found. National conservation status according to Brasil $(2004,2008): \dagger+=$ overexploited, $\dagger=$ endangered. Global conservation status according to IUCN $(2012)$ : $\S=$ least concern, $\$=$ data deficient, - = vulnerable, $*=$ critically endangered, \# = near threatened. (Distribuição Geográfica: CT = circuntropical, TA = Trans-Atlantic, WA = Western Atlantic, $\mathrm{SWA}=$ Southern West Atlantic, SSWA = Southern South West Atlantic, $\mathrm{Ca}=$ Caribbean, $\mathrm{Br}=$ Brazilian Province, $\mathrm{EA}=\mathrm{Eastern} \mathrm{Atlantic}, \mathrm{EP}=\mathrm{Eastern}$ Pacific and $?=$ not found. Status de conservação segundo Brasil $(2004,2008): \dagger \dagger=$ sobreexplotada, $\dagger=$ em perigo. Status de conservação segundo IUCN $(2012)$ : $\S=$ menos preocupante, $\$=$ dados deficientes, $\bullet=$ vulnerável, $*=$ criticamente em perigo, $\#=$ próxima ao perigo). 
Passos, A.C. et al.

Table 1. Continued...

\begin{tabular}{|c|c|c|}
\hline Orders/Families/Species & Geographic distribution & Source \\
\hline A. lepidentostole (Fowler 1911) & $\mathrm{Br}$ & Vendel et al. (2002) \\
\hline Cetengraulis edentulus (Cuvier 1829) & $\mathrm{Ca}+\mathrm{Br}$ & Pichler et al. (2008) \\
\hline Engraulis anchoita Hubbs \& Marini 1935 & SSWA & Ignácio \& Spach (2009) \\
\hline Lycengraulis grossidens (Agassiz 1829) & $\mathrm{Br}+\mathrm{SWA}$ & Pichler et al. (2008) \\
\hline \multicolumn{3}{|l|}{ Pristigasteridae } \\
\hline Pellona harroweri (Fowler 1917) & $\mathrm{Ca}+\mathrm{Br}$ & Spach et al. (2004a) \\
\hline \multicolumn{3}{|l|}{ Siluriformes } \\
\hline \multicolumn{3}{|l|}{ Ariidae } \\
\hline Bagre bagre (Linnaeus 1766) & $\mathrm{Ca}+\mathrm{Br}$ & Schwarz Junior et al. (2007) \\
\hline Cathorops spixii (Agassiz 1829) & $\mathrm{Ca}+\mathrm{Br}$ & Pichler et al. (2008) \\
\hline Genidens barbus (Lacepède 1803) †† & SSWA & Queiroz et al. (2007) \\
\hline G. genidens (Cuvier 1829) & SSWA & Pichler et al. (2008) \\
\hline Notarius luniscutis (Valenciennes 1840) & $\mathrm{Br}$ & Schwarz Junior et al. (2007) \\
\hline \multicolumn{3}{|l|}{ Osmeriformes } \\
\hline \multicolumn{3}{|l|}{ Argentinidae } \\
\hline Glossanodon pygmaeus Cohen 1958 & WA & Godefroid et al. (1999) \\
\hline \multicolumn{3}{|l|}{ Aulopiformes } \\
\hline \multicolumn{3}{|l|}{ Synodontidae } \\
\hline Synodus foetens (Linnaeus 1766) & WA & Pichler et al. (2008) \\
\hline \multicolumn{3}{|l|}{ Gadiformes } \\
\hline \multicolumn{3}{|l|}{ Phycidae } \\
\hline Urophycis brasiliensis (Kaup 1858) & SSWA & Barletta et al. (2008) \\
\hline \multicolumn{3}{|l|}{ Batrachoidiformes } \\
\hline \multicolumn{3}{|l|}{ Batrachoididae } \\
\hline Batrachoides sp. & $?$ & Barletta et al. (2008) \\
\hline Opsanus beta (Goode \& Bean 1880) & $\mathrm{Ca}$ & Ignácio \& Spach (2009) \\
\hline Porichthys porosissimus (Cuvier 1829) & SSWA & Oliveira Neto et al. (2008) \\
\hline \multicolumn{3}{|l|}{ Lophiiformes } \\
\hline \multicolumn{3}{|l|}{ Ogcocephalidae } \\
\hline Ogcocephalus vespertilio (Linnaeus 1758) & $\mathrm{Ca}+\mathrm{SWA}$ & Pinheiro (1999) \\
\hline \multicolumn{3}{|l|}{ Gobiesociformes } \\
\hline \multicolumn{3}{|l|}{ Gobiesocidae } \\
\hline Gobiesox strumosus Cope 1870 & WA & Godefroid et al. (1997) \\
\hline \multicolumn{3}{|l|}{ Atheriniformes } \\
\hline \multicolumn{3}{|l|}{ Atherinopsidae } \\
\hline Atherinella brasiliensis (Quoy \& Gaimard 1825) & $\mathrm{Ca}+\mathrm{Br}$ & Pichler et al. (2008) \\
\hline Membras dissimilis (Carvalho 1956) & SSWA & Godefroid et al. (1999) \\
\hline Odontesthes bonariensis (Valenciennes 1835) & SSWA & Spach et al. (2004a) \\
\hline O. incisa (Jenyns 1841) & SSWA & Spach et al. (2004a) \\
\hline \multicolumn{3}{|l|}{ Cyprinodontiformes } \\
\hline \multicolumn{3}{|l|}{ Poeciliidae } \\
\hline Poecilia vivipara Bloch \& Schneider 1801 & $\mathrm{Br}+\mathrm{SWA}$ & Spach et al. (2004a) \\
\hline \multicolumn{3}{|l|}{ Beloniformes } \\
\hline \multicolumn{3}{|l|}{ Belonidae } \\
\hline Strongylura marina (Walbaum 1792) & WA & Pichler et al. (2008) \\
\hline S. timucu (Walbaum 1792) & WA & Pichler et al. (2008) \\
\hline \multicolumn{3}{|l|}{ Hemiramphidae } \\
\hline Hemiramphus brasiliensis (Linnaeus 1758) & TA & Oliveira Neto et al. (2008) \\
\hline Hyporhamphus roberti (Valenciennes 1847) & $\mathrm{WA}+\mathrm{EP}$ & Pichler (2009) \\
\hline
\end{tabular}

Geographic distribution: $\mathrm{CT}=$ circuntropical, $\mathrm{TA}=$ Trans-Atlantic, WA $=$ Western Atlantic, $\mathrm{SWA}=$ Southern West Atlantic, SSWA $=$ Southern South West Atlantic, $\mathrm{Ca}=$ Caribbean, $\mathrm{Br}=$ Brazilian Province, $\mathrm{EA}=$ Eastern Atlantic, $\mathrm{EP}=$ Eastern Pacific and $?=$ not found. National conservation status according to Brasil $(2004,2008): \dagger=$ overexploited, $\dagger=$ endangered. Global conservation status according to IUCN (2012): $\S=$ least concern, $\$=$ data deficient, • = vulnerable, * = critically endangered, \# = near threatened. (Distribuição Geográfica: CT = circuntropical, TA = Trans-Atlantic, WA = Western Atlantic, $\mathrm{SWA}=$ Southern West Atlantic, $\mathrm{SSWA}=$ Southern South West Atlantic, $\mathrm{Ca}=$ Caribbean, $\mathrm{Br}=$ Brazilian Province, $\mathrm{EA}=\mathrm{Eastern} \mathrm{Atlantic}, \mathrm{EP}=\mathrm{Eastern} \mathrm{Pacific}$ and $?=$ not found. Status de conservação segundo Brasil $(2004,2008): \dagger \dagger=$ sobreexplotada, $\uparrow=$ em perigo. Status de conservação segundo IUCN (2012): $\S=$ menos preocupante, $\ddagger=$ dados deficientes, $\bullet=$ vulnerável, $*=$ criticamente em perigo, $\#=$ próxima ao perigo). 
Table 1. Continued..

\begin{tabular}{|c|c|c|}
\hline Orders/Families/Species & Geographic distribution & Source \\
\hline H. unifasciatus (Ranzani 1841) & $\mathrm{WA}+\mathrm{EP}$ & Pichler et al. (2008) \\
\hline \multicolumn{3}{|l|}{ Exocoetidae } \\
\hline Parexocoetus brachypterus (Richardson 1846) & $\mathrm{TA}+\mathrm{EP}$ & Spach et al. (2004a) \\
\hline \multicolumn{3}{|l|}{ Syngnathiformes } \\
\hline \multicolumn{3}{|l|}{ Fistulariidae } \\
\hline Fistularia petimba Lacepède 1803 & TA & Pichler et al. (2008) \\
\hline F. tabacaria Linnaeus 1758 & TA & Spach et al. (2004a) \\
\hline \multicolumn{3}{|l|}{ Syngnathidae } \\
\hline Bryx dunckeri (Metzelaar 1919) & WA & Spach et al. (2004a) \\
\hline Cosmocampus elucens (Poey 1868) & WA & Spach et al. (2004a) \\
\hline Hippocampus reidi Ginsburg $1933+\uparrow / \$$ & WA & Spach et al. (2004a) \\
\hline Pseudophallus mindii (Meek \& Hildebrand 1923) & $\mathrm{Ca}+\mathrm{Br}$ & Spach et al. (2004a) \\
\hline Syngnathus folletti Herald 1942 & SWA & Spach et al. (2004a) \\
\hline S. pelagicus Linnaeus 1758 & WA & Pichler et al. (2008) \\
\hline \multicolumn{3}{|l|}{ Scorpaeniformes } \\
\hline \multicolumn{3}{|l|}{ Scorpaenidae } \\
\hline Pontinus rathbuni Goode \& Bean 1896 & WA & Spach et al. (2004a) \\
\hline Scorpaena brasiliensis Cuvier 1829 & WA & Nakayama (2004) \\
\hline S. isthmensis Meek \& Hildebrand 1928 & WA & Spach et al. (2007) \\
\hline S.plumieri Bloch 1789 & WA & Pinheiro (1999) \\
\hline \multicolumn{3}{|l|}{ Dactylopteridae } \\
\hline Dactylopterus volitans (Linnaeus 1758) & TA & Spach et al. (2004a) \\
\hline \multicolumn{3}{|l|}{ Triglidae } \\
\hline Prionotus nudigula Ginsburg 1950 & SSWA & Queiroz et al. (2007) \\
\hline P. punctatus (Bloch 1793) & $\mathrm{Ca}+\mathrm{SWA}$ & Spach et al. (2004a) \\
\hline \multicolumn{3}{|l|}{ Perciformes } \\
\hline \multicolumn{3}{|l|}{ Centropomidae } \\
\hline Centropomus parallelus Poey 1860 & WA & Pichler et al. (2008) \\
\hline C.undecimalis (Bloch 1792) & WA & Spach et al. (2004a) \\
\hline \multicolumn{3}{|l|}{ Acropomatidae } \\
\hline Synagrops bellus (Goode \& Bean 1896) & TA & Spach et al. (2004a) \\
\hline \multicolumn{3}{|l|}{ Serranidae } \\
\hline Acanthistius brasilianus (Cuvier 1828) & SSWA & Fávaro (2004) \\
\hline Alphestes afer (Bloch 1793) § & TA & Spach et al. (2004a) \\
\hline Diplectrum radiale (Quoy \& Gaimard 1824) & WA & Spach et al. (2004a) \\
\hline Epinephelus itajara (Lichtenstein 1822) $\dagger \uparrow / *$ & WA & Barletta et al. (2008) \\
\hline Hyporthodus nigritus (Holbrook 1855) * & WA & Godefroid et al. (1997) \\
\hline Mycteroperca bonaci (Poey 1860) $\uparrow \uparrow / \#$ & WA & Fávaro (2004) \\
\hline M. rubra (Bloch 1793) § & TA & Spach et al. (2004a) \\
\hline Rypticus randalli Courtenay 1967 & $\mathrm{Ca}+\mathrm{Br}$ & Spach et al. (2004a) \\
\hline \multicolumn{3}{|l|}{ Pomatomidae } \\
\hline Pomatomus saltatrix (Linnaeus 1766) $\dagger \dagger$ & $\mathrm{CT}$ & Spach et al. (2004a) \\
\hline \multicolumn{3}{|l|}{ Carangidae } \\
\hline Carangoides bartholomaei Cuvier 1833 & WA & Barletta et al. (2008) \\
\hline Caranx hippos (Linnaeus 1766) & TA & Oliveira Neto et al. (2008) \\
\hline C. latus Agassiz 1831 & TA & Pichler et al. (2008) \\
\hline C. ruber (Bloch 1793) & WA & Spach et al. (2004a) \\
\hline Chloroscombrus chrysurus (Linnaeus 1766) & TA & Pichler et al. (2008) \\
\hline
\end{tabular}

Geographic distribution: $\mathrm{CT}=$ circuntropical, $\mathrm{TA}=$ Trans-Atlantic, WA $=$ Western Atlantic, $\mathrm{SWA}=$ Southern West Atlantic, $\mathrm{SSWA}=$ Southern South West Atlantic, $\mathrm{Ca}=$ Caribbean, $\mathrm{Br}=$ Brazilian Province, $\mathrm{EA}=$ Eastern Atlantic, $\mathrm{EP}=$ Eastern Pacific and $?=$ not found. National conservation status according to Brasil $(2004,2008): \dagger \dagger=$ overexploited, $\dagger=$ endangered. Global conservation status according to IUCN (2012): $\S=$ least concern, $\$=$ data deficient, $\cdot=$ vulnerable, $*=$ critically endangered, $\#=$ near threatened. (Distribuição Geográfica: $\mathrm{CT}=$ circuntropical, TA $=$ Trans-Atlantic, WA = Western Atlantic, $\mathrm{SWA}=$ Southern West Atlantic, SSWA $=$ Southern South West Atlantic, $\mathrm{Ca}=$ Caribbean, $\mathrm{Br}=$ Brazilian Province, $\mathrm{EA}=\mathrm{Eastern} \mathrm{Atlantic}, \mathrm{EP}=\mathrm{Eastern}$ Pacific and $?=$ not found. Status de conservação segundo Brasil $(2004,2008): \dagger \dagger=$ sobreexplotada, $\dagger=$ em perigo. Status de conservação segundo IUCN (2012): $\S=$ menos preocupante, $\$=$ dados deficientes, $\bullet=$ vulnerável, $*$ = criticamente em perigo, \# = próxima ao perigo). 
Table 1. Continued...

\begin{tabular}{|c|c|c|}
\hline Orders/Families/Species & Geographic distribution & Source \\
\hline Hemicaranx amblyrhynchus (Cuvier 1833) & WA & Corrêa et al. (1986) \\
\hline Oligoplites palometa (Cuvier 1832) & $\mathrm{Ca}+\mathrm{Br}$ & Barletta et al. (2008) \\
\hline O. saliens (Bloch 1793) & $\mathrm{Ca}+\mathrm{SWA}$ & Pichler et al. (2008) \\
\hline O. saurus (Bloch \& Schneider 1801) & WA & Spach et al. (2004a) \\
\hline Selene setapinnis (Mitchill 1815) & WA & Spach et al. (2004a) \\
\hline S. vomer (Linnaeus 1758) & WA & Pichler et al. (2008) \\
\hline Seriola lalandi Valenciennes 1833 & $\mathrm{CT}$ & Spach et al. (2004a) \\
\hline Trachinotus carolinus (Linnaeus 1766) & WA & Pichler et al. (2008) \\
\hline T. falcatus (Linnaeus 1758) & WA & Pichler et al. (2008) \\
\hline T. goodei Jordan \& Evermann 1896 & WA & Spach et al. (2004a) \\
\hline T. marginatus Cuvier 1832 & SSWA & Spach et al. (2004a) \\
\hline Uraspis secunda (Poey 1860) & CT & Godefroid et al. (1997) \\
\hline \multicolumn{3}{|l|}{ Lutjanidae } \\
\hline Lutjanus analis (Cuvier 1828) $\uparrow \uparrow / \bullet$ & WA & Spach et al. (2004a) \\
\hline L. griseus (Linnaeus 1758) & WA & Spach et al. (2003) \\
\hline L. synagris (Linnaeus 1758) & WA & Pinheiro (1999) \\
\hline \multicolumn{3}{|l|}{ Lobotidae } \\
\hline Lobotes surinamensis (Bloch 1790) & CT & Godefroid et al. (1997) \\
\hline \multicolumn{3}{|l|}{ Gerreidae } \\
\hline Diapterus auratus Ranzani 1842 & WA & Ignácio \& Spach (2009) \\
\hline Diapterus rhombeus (Cuvier 1829) & $\mathrm{Ca}+\mathrm{Br}$ & Pichler et al. (2008) \\
\hline Eucinostomus argenteus Baird \& Girard 1855 & $\mathrm{WA}+\mathrm{EP}$ & Pichler et al. (2008) \\
\hline E. gula (Quoy \& Gaimard 1824) & WA & Pichler et al. (2008) \\
\hline E. melanopterus (Bleeker 1863) & TA & Pichler et al. (2008) \\
\hline Eugerres brasilianus (Cuvier 1830) & WA & Oliveira Neto et al. (2008) \\
\hline Ulaema lefroyi (Goode 1874) & $\mathrm{Ca}+\mathrm{Br}$ & Spach et al. (2003) \\
\hline \multicolumn{3}{|l|}{ Haemulidae } \\
\hline Anisotremus surinamensis (Bloch 1791) & WA & Pichler et al. (2008) \\
\hline A. virginicus (Linnaeus 1758) & WA & Spach et al. (2004a) \\
\hline Boridia grossidens Cuvier 1830 & SSWA & Spach et al. (2006) \\
\hline Conodon nobilis (Linnaeus 1758) & WA & Spach et al. (2004a) \\
\hline Genyatremus luteus (Bloch 1790) & $\mathrm{Ca}+\mathrm{Br}$ & Spach et al. (2004a) \\
\hline Haemulon steindachneri (Jordan \& Gilbert 1882) & $\mathrm{Ca}+\mathrm{SWA}$ & Godefroid et al. (1997) \\
\hline Orthopristis ruber (Cuvier 1830) & $\mathrm{Ca}+\mathrm{SWA}$ & Pichler et al. (2008) \\
\hline Pomadasys corvinaeformis (Steindachner 1868) & $\mathrm{Ca}+\mathrm{SWA}$ & Spach et al. (2004a) \\
\hline P. ramosus (Poey 1860) & $\mathrm{Ca}+\mathrm{Br}$ & Hackradt et al. (2009) \\
\hline \multicolumn{3}{|l|}{ Sparidae } \\
\hline $\begin{array}{l}\text { Archosargus probatocephalus } \\
\text { (Walbaum 1792) }\end{array}$ & WA & Pichler (2009) \\
\hline $\begin{array}{l}\text { Archosargus rhomboidalis } \\
\text { (Linnaeus 1758) }\end{array}$ & WA & Godefroid et al. (1997) \\
\hline $\begin{array}{l}\text { Calamus penna } \\
\text { (Valenciennes 1830) }\end{array}$ & WA & Pinheiro (1999) \\
\hline $\begin{array}{l}\text { Diplodus argenteus } \\
\text { (Valenciennes 1830) }\end{array}$ & SWA & Spach et al. (2004a) \\
\hline \multicolumn{3}{|l|}{ Sciaenidae } \\
\hline Bairdiella ronchus (Cuvier 1830) & $\mathrm{Ca}+\mathrm{Br}$ & Pichler et al. (2008) \\
\hline Ctenosciaena gracilicirrhus (Metzelaar 1919) & $\mathrm{Ca}+\mathrm{Br}$ & Spach et al. (2004a) \\
\hline Cynoscion acoupa (Lacepède 1801) & $\mathrm{Ca}+\mathrm{SWA}$ & Oliveira Neto et al. (2008) \\
\hline
\end{tabular}

Geographic distribution: $\mathrm{CT}=$ circuntropical, TA $=$ Trans-Atlantic, WA $=$ Western Atlantic, SWA $=$ Southern West Atlantic, SSWA $=$ Southern South West Atlantic, $\mathrm{Ca}=$ Caribbean, $\mathrm{Br}=$ Brazilian Province, $\mathrm{EA}=$ Eastern Atlantic, $\mathrm{EP}=$ Eastern Pacific and ? = not found. National conservation status according to Brasil $(2004,2008): \dagger \dagger=$ overexploited, $\dagger=$ endangered. Global conservation status according to IUCN $(2012)$ : $\S=1$ least concern, $\$=$ data deficient, - = vulnerable, $*=$ critically endangered, \# = near threatened. (Distribuição Geográfica: CT $=$ circuntropical, TA $=$ Trans-Atlantic, WA $=$ Western Atlantic, $\mathrm{SWA}=$ Southern West Atlantic, SSWA = Southern South West Atlantic, $\mathrm{Ca}=$ Caribbean, $\mathrm{Br}=\mathrm{Brazilian}$ Province, EA $=\mathrm{Eastern}$ Atlantic, EP = Eastern Pacific and $?=$ not found. Status de conservação segundo Brasil $(2004,2008): \dagger \dagger=$ sobreexplotada, $\dagger=$ em perigo. Status de conservação segundo IUCN (2012): $\S=$ menos preocupante, $\ddagger=$ dados deficientes, $\bullet=$ vulnerável, $*=$ criticamente em perigo, \# $=$ próxima ao perigo). 
Table 1. Continued..

\begin{tabular}{|c|c|c|}
\hline Orders/Families/Species & Geographic distribution & Source \\
\hline C. jamaicensis (Vaillant \& Bocourt 1883) & $\mathrm{Ca}+\mathrm{SWA}$ & Schwarz Junior et al. (2007) \\
\hline C. leiarchus (Cuvier 1830) & $\mathrm{Ca}+\mathrm{Br}$ & Spach et al. (2004a) \\
\hline C. microlepidotus (Cuvier 1830) & $\mathrm{Br}$ & Spach et al. (2004a) \\
\hline C. striatus (Cuvier 1829) & SSWA & Ignácio \& Spach (2009) \\
\hline C. virescens (Cuvier 1830) & $\mathrm{Ca}+\mathrm{Br}$ & Schwarz Junior et al. (2007) \\
\hline Isopisthus parvipinnis (Cuvier 1830) & $\mathrm{Ca}+\mathrm{Br}$ & Spach et al. (2004a) \\
\hline Larimus breviceps Cuvier 1830 & $\mathrm{Ca}+\mathrm{Br}$ & Spach et al. (2004a) \\
\hline Macrodon atricauda (Bloch \& Schneider 1801) †† & $\mathrm{Br}+\mathrm{SWA}$ & Schwarz Junior et al. (2007) \\
\hline Menticirrhus americanus (Linnaeus 1758) & WA & Pichler et al. (2008) \\
\hline M. littoralis (Holbrook 1847) & WA & Spach et al. (2004a) \\
\hline Micropogonias furnieri (Desmarest 1823) $\dagger \dagger$ & $\mathrm{Ca}+\mathrm{SWA}$ & Pichler et al. (2008) \\
\hline Nebris microps Cuvier 1830 & $\mathrm{Br}$ & Schwarz Junior et al. (2007) \\
\hline Ophioscion punctatissimus Meek \& Hildebrand 1925 & $\mathrm{Ca}+\mathrm{Br}$ & Spach et al. (2004a) \\
\hline Paralonchurus brasiliensis (Steindachner 1875) & $\mathrm{Ca}+\mathrm{SWA}$ & Schwarz Junior et al. (2007) \\
\hline Pogonias cromis (Linnaeus 1766) & WA & Pichler (2009) \\
\hline Stellifer brasiliensis (Schultz 1945) & $\mathrm{Br}$ & Spach et al. (2004a) \\
\hline S. rastrifer (Jordan 1889) & $\mathrm{Br}+\mathrm{SSWA}$ & Ignácio \& Spach (2009) \\
\hline S. stellifer (Bloch 1790) & $\mathrm{Br}$ & Spach et al. (2004a) \\
\hline Umbrina canosai Berg $1895+\dagger$ & SSWA & Spach et al. (2004a) \\
\hline U. coroides Cuvier 1830 & WA & Ignácio \& Spach (2009) \\
\hline \multicolumn{3}{|l|}{ Polynemidae } \\
\hline Polydactylus oligodon (Günther 1860) & WA & Godefroid et al. (1999) \\
\hline P. virginicus (Linnaeus 1758) & WA & Spach et al. (2004a) \\
\hline \multicolumn{3}{|l|}{ Mullidae } \\
\hline Mullus auratus Jordan \& Gilbert 1882 & WA & Barletta et al. (2008) \\
\hline Pseudupeneus maculatus (Bloch 1793) & WA & Spach et al. (2004a) \\
\hline Upeneus parvus Poey 1852 & WA & Pinheiro (1999) \\
\hline \multicolumn{3}{|l|}{ Mugilidae } \\
\hline M. curema Valenciennes 1836 & $\mathrm{TA}+\mathrm{EP}$ & Pichler et al. (2008) \\
\hline M. curvidens Valenciennes 1836 & $\mathrm{Ca}+\mathrm{Br}$ & Spach et al. (2004a) \\
\hline M. incilis Hancock 1830 & $\mathrm{Ca}+\mathrm{Br}$ & Spach et al. (2004a) \\
\hline M. liza Valenciennes $1836 \dagger \dagger$ & WA & Pichler et al. (2008) \\
\hline Mugil sp. & $?$ & Spach et al. (2004a) \\
\hline \multicolumn{3}{|l|}{ Cichlidae } \\
\hline Geophagus brasiliensis (Quoy \& Gaimard 1824) & SSWA & Pichler (2009) \\
\hline Oreochromis niloticus (Linnaeus 1758) & EA & Contente et al.(2010) \\
\hline \multicolumn{3}{|l|}{ Uranoscopidae } \\
\hline Astroscopus sexspinosus (Steindachner 1876) & SSWA & Spach et al. (2004a) \\
\hline A. y-graecum (Cuvier 1829) & WA & Pichler et al. (2008) \\
\hline \multicolumn{3}{|l|}{ Pinguipedidae } \\
\hline Pinguipes brasilianus Cuvier 1829 & SSWA & Spach et al. (2004a) \\
\hline \multicolumn{3}{|l|}{ Clinidae } \\
\hline Ribeiroclinus eigenmanni (Jordan 1888) & SSWA & Spach et al. (2004a) \\
\hline \multicolumn{3}{|l|}{ Blenniidae } \\
\hline Hypleurochilus sp. & $?$ & Corrêa et al. (1986) \\
\hline Parablennius pilicornis (Cuvier 1829) & TA & Spach et al. (2004b) \\
\hline \multicolumn{3}{|l|}{ Eleotridae } \\
\hline Dormitator maculatus (Bloch 1792) & WA & Queiroz et al. (2007) \\
\hline
\end{tabular}

Geographic distribution: $\mathrm{CT}=$ circuntropical, $\mathrm{TA}=$ Trans-Atlantic, WA $=$ Western Atlantic, $\mathrm{SWA}=$ Southern West Atlantic, $\mathrm{SSWA}=$ Southern South West Atlantic, $\mathrm{Ca}=$ Caribbean, $\mathrm{Br}=$ Brazilian Province, $\mathrm{EA}=$ Eastern Atlantic, $\mathrm{EP}=$ Eastern Pacific and $?=$ not found. National conservation status according to Brasil $(2004,2008): \dagger=$ overexploited, $\dagger=$ endangered. Global conservation status according to IUCN (2012): $\S=$ least concern, $\$=$ data deficient, $\cdot=$ vulnerable, $*=$ critically endangered, $\#=$ near threatened. (Distribuição Geográfica: $\mathrm{CT}=$ circuntropical, TA $=$ Trans-Atlantic, WA = Western Atlantic, $\mathrm{SWA}=$ Southern West Atlantic, SSWA $=$ Southern South West Atlantic, $\mathrm{Ca}=$ Caribbean, $\mathrm{Br}=$ Brazilian Province, EA $=\mathrm{Eastern} \mathrm{Atlantic}, \mathrm{EP}=\mathrm{Eastern}$ Pacific and $?=$ not found. Status de conservação segundo Brasil $(2004,2008): \dagger=$ sobreexplotada, $\dagger=$ em perigo. Status de conservação segundo IUCN $(2012)$ : $\S=$ menos preocupante, $\$=$ dados deficientes, $\bullet=$ vulnerável, $*$ = criticamente em perigo, $\#=$ próxima ao perigo). 
Table 1. Continued...

\begin{tabular}{|c|c|c|}
\hline Orders/Families/Species & Geographic distribution & Source \\
\hline Eleotris pisonis (Gmelin 1789) & WA & Fávaro (2004) \\
\hline Guavina guavina (Valenciennes 1837) & WA & Pichler et al. (2008) \\
\hline \multicolumn{3}{|l|}{ Gobiidae } \\
\hline Awaous tajasica (Lichtenstein 1822) & WA & Vendel et al. (2002) \\
\hline Bathygobius soporator (Valenciennes 1837) & TA & Pichler et al. (2008) \\
\hline Ctenogobius boleosoma (Jordan \& Gilbert 1882) & WA & Pichler et al. (2008) \\
\hline C. shufeldti (Jordan \& Eigenmann 1887) & WA & Pichler et al. (2008) \\
\hline C. smaragdus (Valenciennes 1837) & WA & Pichler et al. (2008) \\
\hline C. stigmaticus (Poey 1860) & WA & Vendel et al. (2002) \\
\hline Gobioides broussonnetii Lacepède 1800 & WA & Cortellete et al. (2009) \\
\hline Gobionellus oceanicus (Pallas 1770) & WA & Pichler et al. (2008) \\
\hline Microgobius meeki Evermann \& Marsh 1899 & $\mathrm{Ca}+\mathrm{Br}$ & Pichler et al. (2008) \\
\hline \multicolumn{3}{|l|}{ Ephippidae } \\
\hline Chaetodipterus faber (Broussonet 1782) & WA & Pichler et al. (2008) \\
\hline \multicolumn{3}{|l|}{ Sphyraenidae } \\
\hline Sphyraena barracuda (Edwards 1771) & CT & Spach et al. (2004a) \\
\hline S. guachancho Cuvier 1829 & TA & Spach et al. (2004a) \\
\hline S. picudilla Poey 1860 & WA & Abilhôa (1998) \\
\hline S. tome Fowler 1903 & SSWA & Vendel et al. (2003) \\
\hline \multicolumn{3}{|l|}{ Trichiuridae } \\
\hline Trichiurus lepturus Linnaeus 1758 & $\mathrm{CT}$ & Spach et al. (2004a) \\
\hline \multicolumn{3}{|l|}{ Scombridae } \\
\hline Acanthocybium solandri (Cuvier 1832) § & CT & Fávaro (2004) \\
\hline $\begin{array}{l}\text { Scomberomorus brasiliensis Collette, Russo \& Zavala-Camin } \\
1978 \S\end{array}$ & $\mathrm{Ca}+\mathrm{Br}$ & Pichler et al. (2008) \\
\hline S. cavalla (Cuvier 1829) $\S$ & WA & Spach et al. (2004a) \\
\hline \multicolumn{3}{|l|}{ Stromateidae } \\
\hline Peprilus paru (Linnaeus 1758) & WA & Spach et al. (2004a) \\
\hline \multicolumn{3}{|l|}{ Pleuronectiformes } \\
\hline \multicolumn{3}{|l|}{ Paralichthyidae } \\
\hline Citharichthys arenaceus Evermann \& Marsh 1900 & WA & Pichler et al. (2008) \\
\hline C. macrops Dresel 1885 & WA & Félix et al. (2007) \\
\hline C. spilopterus Günther 1862 & WA & Pichler et al. (2008) \\
\hline Etropus crossotus Jordan \& Gilbert 1882 & $\mathrm{WA}+\mathrm{EP}$ & Pichler et al. (2008) \\
\hline Paralichthys brasiliensis (Ranzani 1842) & SWA & Pichler et al. (2008) \\
\hline P. orbignyanus (Valenciennes 1839) & SSWA & Pichler et al. (2008) \\
\hline P. patagonicus Jordan 1889 & SSWA+EP & Stefanoni (2007) \\
\hline P. tropicus Ginsburg 1933 & WA & Santos et al. (2002) \\
\hline Syacium papillosum (Linnaeus 1758) & WA & Félix et al. (2007) \\
\hline \multicolumn{3}{|l|}{ Pleuronectidae } \\
\hline Oncopterus darwinii Steindachner 1874 & SSWA & Godefroid et al. (1997) \\
\hline Pleuronectes sp. & $?$ & Barletta et al. (2008) \\
\hline \multicolumn{3}{|l|}{ Achiridae } \\
\hline Achirus declivis Chabanaud 1940 & WA & Ignácio \& Spach (2009) \\
\hline A. lineatus (Linnaeus 1758) & WA & Pichler et al. (2008) \\
\hline
\end{tabular}

Geographic distribution: $\mathrm{CT}=$ circuntropical, $\mathrm{TA}=$ Trans-Atlantic, WA $=$ Western Atlantic, SWA $=$ Southern West Atlantic, SSWA $=$ Southern South West Atlantic, $\mathrm{Ca}=$ Caribbean, $\mathrm{Br}=$ Brazilian Province, $\mathrm{EA}=$ Eastern Atlantic, $\mathrm{EP}=$ Eastern Pacific and $?=$ not found. National conservation status according to Brasil $(2004,2008): \dagger \dagger=$ overexploited, $\dagger=$ endangered. Global conservation status according to IUCN (2012): $\S=$ least concern, $\$=$ data deficient, - = vulnerable, $*=$ critically endangered, \# = near threatened. (Distribuição Geográfica: $\mathrm{CT}=$ circuntropical, TA $=$ Trans-Atlantic, WA $=$ Western Atlantic, $\mathrm{SWA}=$ Southern West Atlantic, SSWA = Southern South West Atlantic, $\mathrm{Ca}=$ Caribbean, $\mathrm{Br}=$ Brazilian Province, EA = Eastern Atlantic, EP = Eastern Pacific and $?=$ not found. Status de conservação segundo Brasil $(2004,2008): \dagger \dagger=$ sobreexplotada, $\dagger=$ em perigo. Status de conservação segundo IUCN $(2012)$ : $\S=$ menos preocupante, $\$=$ dados deficientes, $\bullet=$ vulnerável, $*=$ criticamente em perigo, $\#=$ próxima ao perigo). 
Table 1. Continued..

\begin{tabular}{|c|c|c|}
\hline Orders/Families/Species & Geographic distribution & Source \\
\hline Trinectes microphthalmus (Chabanaud 1928) & $\mathrm{Ca}+\mathrm{Br}$ & Fávaro (2004) \\
\hline T. paulistanus (Miranda Ribeiro 1915) & $\mathrm{Ca}+\mathrm{Br}$ & Spach et al. (2004a) \\
\hline \multicolumn{3}{|l|}{ Cynoglossidae } \\
\hline Symphurus plagusia (Bloch \& Schneider 1801) & $\mathrm{Ca}+\mathrm{Br}$ & Spach et al. (2004a) \\
\hline S. tesselatus (Quoy \& Gaimard 1824) & $\mathrm{Ca}+\mathrm{Br}+\mathrm{SSWA}$ & Spach et al. (2004a) \\
\hline \multicolumn{3}{|l|}{ Tetraodontiformes } \\
\hline \multicolumn{3}{|l|}{ Monacanthidae } \\
\hline Stephanolepis hispidus (Linnaeus 1766) & TA & Spach et al. (2004a) \\
\hline \multicolumn{3}{|l|}{ Tetraodontidae } \\
\hline Lagocephalus laevigatus (Linnaeus 1766) & TA & Pichler et al. (2008) \\
\hline Sphoeroides greeleyi Gilbert 1900 & $\mathrm{Ca}+\mathrm{Br}$ & Spach et al. (2004a) \\
\hline S. spengleri (Bloch 1785) & TA & Schwarz Junior et al. (2007) \\
\hline S. testudineus (Linnaeus 1758) & WA & Pichler et al. (2008) \\
\hline S. tyleri Shipp 1972 & $\mathrm{Br}$ & Vendel et al. (2002) \\
\hline \multicolumn{3}{|l|}{ Diodontidae } \\
\hline Chilomycterus spinosus (Linnaeus 1758) & SWA & Pichler et al. (2008) \\
\hline
\end{tabular}

Geographic distribution: $\mathrm{CT}=$ circuntropical, $\mathrm{TA}=$ Trans-Atlantic, WA = Western Atlantic, SWA = Southern West Atlantic, SSWA = Southern South West Atlantic, $\mathrm{Ca}=$ Caribbean, $\mathrm{Br}=$ Brazilian Province, $\mathrm{EA}=$ Eastern Atlantic, $\mathrm{EP}=$ Eastern Pacific and $?=$ not found. National conservation status according to Brasil $(2004,2008): \dagger \dagger=$ overexploited, $\dagger=$ endangered. Global conservation status according to IUCN (2012): $\S=$ least concern, $\ddagger=$ data deficient, - = vulnerable, $*=$ critically endangered, $\#=$ near threatened. (Distribuição Geográfica: $\mathrm{CT}=$ circuntropical, $\mathrm{TA}=$ Trans-Atlantic, WA $=$ Western Atlantic, $\mathrm{SWA}=$ Southern West Atlantic, SSWA $=$ Southern South West Atlantic, $\mathrm{Ca}=$ Caribbean, $\mathrm{Br}=$ Brazilian Province, EA $=$ Eastern Atlantic, $\mathrm{EP}=\mathrm{Eastern}$ Pacific and $?=$ not found. Status de conservação segundo Brasil $(2004,2008): \dagger \dagger=$ sobreexplotada, $\dagger=$ em perigo. Status de conservação segundo IUCN $(2012)$ : $\S=$ menos preocupante, $\$=$ dados deficientes, $\bullet=$ vulnerável, $*$ = criticamente em perigo, \# = próxima ao perigo).

and/or the use of different sampling gears across tropical estuaries around the world (Blaber 2002).

The species' classification into the geographical categories used in this study present here, which were adapted from Floeter et al. (2008) and Luiz Junior et al. (2008), fits well with the species' distribution described on the literature. This is an indicative of utility of such a classification for future studies aiming to classify SW Atlantic estuarine fishes. Most species in the PEC are widely distributed throughout the Western Atlantic (40\%), followed by those that are restricted to (and occur both in) the Caribbean and Brazilian (15\%), and South-West Atlantic (11\%) provinces (Figure 2). Thus, the PEC fish fauna can be regarded as a mixture between the fish fauna typical from the tropical Brazilian coast and those with affinities of temperate Argentinean and Uruguayan zones.

Our results suggest an expansion of the geographical distribution for G. pygmaeus and M. auratus. G. pygmaeus, recorded in Godefroid et al. (1997, 1999), which was previously reported only for the tropical Western Atlantic, from South Carolina state to the tropical coast of Brazil, near the equator (Eschmeyer 2010), and Mullus auratus, recorded by Barletta et al. (2008), previously reported from North Carolina, USA, to the Caribbean (Floeter et al. 2008).

Only a minor part $(8 \%)$ of the PEC fish fauna was evaluated as regards the conservation status: ten are on the IUCN Red List (International... 2012), 12 on the ME list (Brasil 2004, 2008), and four are on both. Of the ten species on the IUCN Red List (International... 2012), two (Epinephelus itajara and Hyporthodus nigritus) are critically endangered, one (Mycteroperca bonaci) is near threatened, one (Lutjanus analis) is vulnerable, five (Alphestes afer, Mycteroperca rubra, Acanthocybium solandri, Scomberomorus brasiliensis and S. cavalla) are least concern and one (Hippocampus reidi) is data deficient. Among the 12 species on the Ministry of the Environment List, 11 are overexploited and one is endangered (Rhinobatus horkelii). Many fishery species in PEC are found to be classified as threatened on the list for endangered fauna of the adjacent state of São Paulo (São Paulo 2010). It is supposed that many species in PEC have similar conservation status 'threatened' as face similar threats to those of São Paulo state coast, where the fishing pressure is similar to that of Paraná state. Such a setting reinforces the urgent need for critical, basic information for fish species to support their conservation effort through IUCN classification in this important estuary.

Particular concern must be place to introduction in the PEC of the species Opsanus beta, from the North Atlantic (Eschmeyer 2010), and Oreochromis niloticus from Africa. Although the impact of $O$. beta on the native fish fauna is still unknown (Caires et al. 2007), it is supposed to be serious, as it is a voracious, generalized predator (Gray \& Winn 1961). Moreover, recent field observations have already reported $O$. beta as a very common by-catch in long-line inside estuary, thus affecting local fisheries. O. niloticus is classified as a pest and has been reported to adversely impact ecosystem after its introduction (Froese \& Pauly 2010). Future studies assessing the degree of establishment success of such species and their impact on the local fish biodiversity are strongly recommended.

This study provides a full list of fish species of the Paranaguá Estuarine Complex based on a robust dataset, which takes into account a wide and representative spatio temporal variability, largely improving the species detectability. The ichthyofauna of the PEC contains taxa with affinities from the tropical Brazilian coast and those with affinities of temperate Argentinean and Uruguayan waters. The fish richness of the system of 213 species is surprisingly higher than other systems in Brazil and around world, which emphasize the importance of the region for global biodiversity conservation. Once we have knowledge of the species and the richness of PEC, we suggest a fauna monitoring for a regional analysis of conservation status and more details about impacts of exotic species for the creation of management and conservation proposals. 
Passos, A.C. et al.

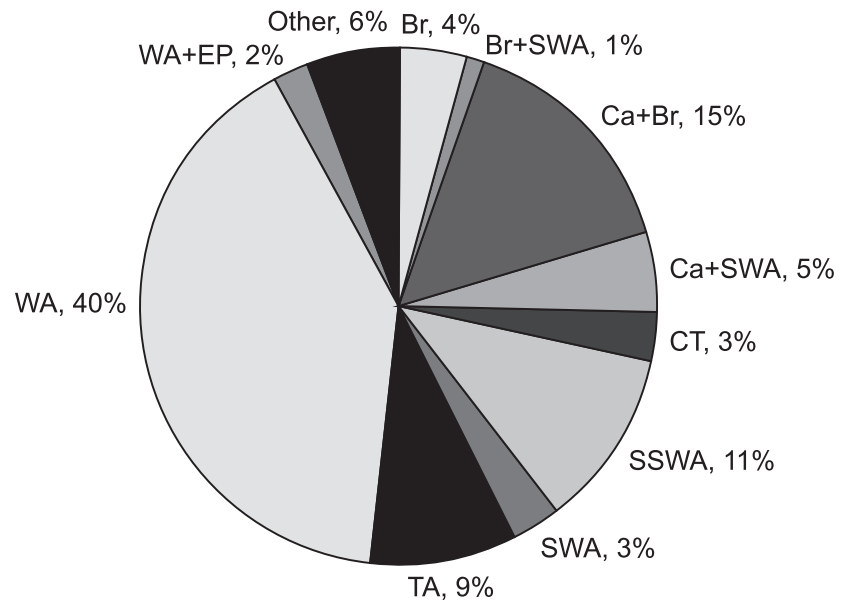

Figure 2. Geographic distribution of the ichthyofauna recorded in the Paranaguá Estuarine Complex, Southwest Atlantic (CT = circuntropical, $\mathrm{TA}=$ Trans-Atlantic, $\mathrm{WA}=$ Western Atlantic, $\mathrm{SWA}=$ Southern West Atlantic, $\mathrm{SSWA}=$ Southern South West Atlantic, $\mathrm{Ca}=$ Caribbean, $\mathrm{Br}=$ Brazilian Province, $\mathrm{EA}=$ Eastern Atlantic, $\mathrm{EP}=$ Eastern Pacific and Other $=$ categories (Trans-Atlantic + Eastern Pacific, Brazilian Province + southern South-West Atlantic, Caribbean, Caribbean + Brazilian Province + Eastern Pacific, Caribbean + Brazilian Province + southern South-West Atlantic, southern South-West Atlantic + Eastern Pacific, Eastern Atlantic) that represent less than $1 \%$ each).

Figura 2. Distribuição Geográfica da ictiofauna encontrada no Complexo Estuarino de Paranaguá, Atlântico Sul $(\mathrm{CT}=$ circuntropical, $\mathrm{TA}=$ TransAtlantic, WA $=$ Western Atlantic, $\mathrm{SWA}=$ Southern West Atlantic, $\mathrm{SSWA}=$ Southern South West Atlantic, $\mathrm{Ca}=$ Caribbean, $\mathrm{Br}=$ Brazilian Province, EA = Eastern Atlantic, EP = Eastern Pacific e Outros $=$ categorias (Trans-Atlantic + Eastern Pacific, Brazilian Province + southern South-West Atlantic, Caribbean, Caribbean + Brazilian Province + Eastern Pacific, Caribbean + Brazilian Province + southern South-West Atlantic, southern South-West Atlantic + Eastern Pacific, Eastern Atlantic) que representam menos de $1 \%$ cada.

\section{Acknowledgements}

We would like to thank the CEM Geological Oceanography Laboratory for the map data base and Pâmela Emanuelly Cattani for their valuable help with the map. Funding was provided by CAPES foundation through a master grant to Ana Carolina dos Passos.

\section{References}

ABILHÔA, V. 1998. Composição e estrutura da ictiofauna em um banco areno-lodoso na Ilha do Mel, Paraná, Brasil. Dissertação de mestrado, Universidade Federal do Paraná, Curitiba.

ALCÂNTARA, A.V. 2006. A ictiofauna do estuário do Rio Sergipe. In Rio Sergipe: importância, vulnerabilidade e preservação (J.P.H. Alves, org.). Editora UFS, Sergipe, p.111-142.

ANDRADE-TUBINO, M.F., RIBEIRO, A.L.R. \& VIANNA, M. 2008. Organização espaço-temporal das ictiocenoses demersais nos ecossistemas estuarinos brasileiros: uma síntese. Oecol. Bras. 12(4):640-661.

BARLETTA, M., BARLETTA-BERGAN, A., SAINT-PAUL, U. \& HUBOLD, G. 2005. The role of salinity in structuring the fish assemblages in a tropical estuary. J. Fish. Biol. 66:45-72. http://dx.doi.org/10.1111/ j.0022-1112.2005.00582.x

BARLETTA, M. \& BLABER, S.J.M. 2007. Comparision of fish assemblages and guilds in tropical habitats of the Embley (Indo-West Pacific) and Caeté (Western Atlantic) estuaries. Bull. Mar. Sci. 80(3):647-680.
BARLETTA, M., AMARAL, C.S., CORRÊA, M.F.M., GUEBERT, F., DANTAS, D.V., LORENZI, L. \& SAINT-PAUL, U. 2008. Factors affecting seasonal variations in demersal fish assemblages at an ecocline in a tropical-subtropical estuary. J. Fish Biol. 73:1314-1336. http://dx.doi. org/10.1111/j.1095-8649.2008.02005.x

BARLETTA, M., JAUREGUIZAR, A.J., BAIGUN, C., FONTOURA, N.F., AGOSTINHO, A.A., ALMEIDA-VAL, V.M.F., VAL, A.L., TORRES, R.A., JIMENES-SEGURA, L.F., GIARRIZZO, T., FABRÉ, N.N., BATISTA, V.S., LASSO, C., TAPHORN, D.C., COSTA, M.F., CHAVES, P.T., VIEIRA, J.P. \& CORRÊA, M.F.M. 2010. Fish and aquatic habitat conservation in South America: a continental overview with emphasis on neotropical systems. J. Fish. Biol. 76(9):2118-2176. PMid:20557657. http://dx.doi.org/10.1111/j.1095-8649.2010.02684.x

BLABER, S.J.M. 2002. Fish in hot water: the challenges facing fish and fisheries research in tropical estuaries. J. Fish Biol. 61(sA):1-20. http:// dx.doi.org/10.1111/j.1095-8649.2002.tb01757.x

BLABER, S.J.M., BREWER, D.T., SALINI, J.P. \& KERR, J. 1990. Biomass, catch rates and abundances of demersal fishes, particularly predators of prawns, in a tropical bay in the Gulf of Carpentaria, Australia. Mar. Biol. 107:397-408.

BRASIL. Ministério do Meio Ambiente - MMA. 2004. Instrução Normativa $\mathrm{n}^{\circ}$ 005, de 21 de maio de 2004. Lista Nacional das Espécies de Invertebrados Aquáticos e Peixes Ameaçadas de Extinção. Diário Oficial da República Federativa do Brasil, Brasília, DF, 12 abr. 2004. http://www. mma.gov.br/estruturas/179/_arquivos/179_05122008033927.pdf. (último acesso em 19/01/2011).

BRASIL. Ministério do Meio Ambiente - MMA. 2008. Livro vermelho da fauna Brasileira ameaçada de extinção. Ministério do Meio Ambiente, Brasília, v.2.

CAIRES, R.A., PICHLER, H.A., SPACH, H.L. \& IGNÁCIO, J.M. 2007. Opsanus brasiliensis Rotundo, Spinelli \& Zavalla-Camin, 2005 (Teleostei: Batrachoidiformes: Batrachoididae), sinônimo-júnior de Opsanus beta (Goode \& Bean, 1880), com notas sobre a ocorrência da espécie na costa brasileira. Biota Neotrop. 7(2): http://www.biotaneotropica.org.br/v7n2/ pt/fullpaper?bn02307022007+pt (último acesso em 20/01/2011).

CARVALHO-FILHO, A., SANTOS, S. \& SAMPAIO, I. 2010. Macrodon atricauda (Günther, 1880) (Perciformes: Sciaenidae), a valid species from the southwestern Atlantic, with comments on its conservation. Zootaxa 2519:48-58.

CHAVES, P.T. \& CORRÊA, M.F.M. 1998. Composição ictiofaunística da área de manguezal da Baía de Guaratuba, Estado do Paraná, Brasil. Rev. Bras. Zool. 15(1):195-202. http://dx.doi.org/10.1590/S010181751998000100017

CHAVES, P.T. \& VENDEL, A.L. 2001. Nota complementar sobre a composição ictiofaunística da Baía de Guaratuba, Paraná, Brasil. Rev. Bras. Zool. 18(1):349-352. http://dx.doi.org/10.1590/S010181752001000500032

CONTENTE, R.F., STEFANONI, M.F. \& SPACH, H.L. 2011. Fish assemblage structure in an estuary of the Atlantic Forest biodiversity hotspot (southern Brazil). Ichthiol. Res. 58(1):38-50. http://dx.doi. org/10.1007/s10228-010-0192-0

CORRÊA, M.F.M., CORDEIRO, A.A.M. \& JUSTI, I.M. 1986. Catálogo dos peixes marinhos da Coleção da Divisão de Zoologia e Geologia da Prefeitura Municipal de Curitiba - I. Nerítica 1(1):1-83.

CORTELLETE, G.M., GODEFROID, R.S., SILVA, A.L.C., CATTANII, A.P., DAROS, F.A. \& SPACH, H.L. 2009. Peixes da área de deposição de material dragado na Baía de Antonina, Paraná, Brasil. Cad. Esc. Saúde - Cienc. Biol. Unibrasil 02:1-19.

CRAIG, M.T. \& HASTINGS, P.A. 2007. A molecular phylogeny of the groupers of the subfamily Epinephelinae (Serranidae) with a revised classification of Epinephelini. Ichthiol. Res. 54:1-17. http://dx.doi. org/10.1007/s10228-006-0367-x

DIAS, J.F., GONÇALVES, A.M., FERNANDEZ, W.S., SILBIGER, H.L.N., FIADI, C.B. \& SCHMIDT, T.C.S. 2011. Ichthyofauna in an estuary of the Mataripe area, Todos os Santos Bay, Bahia, Brazil. Braz. J. Oceanogr. 59(1):75-95. http://dx.doi.org/10.1590/S167987592011000100006 
DIEGUES, A.C. 1995. The Mata Atlantica Biosphere Reserve: an overview (Brazil). South-South Cooperation Programme on Environmentally Sound Socio-Economic Development in the Humid Tropics. UNESCO, Paris.

ESCHMEYER, W.N. 2010. Catalog of Fishes. California Academy of Sciences, San Francisco. http://www.calacademy.org/research/ ichthyology/catalog/ (último acesso em 20/10/2010).

FÁVARO, L.F. 2004. A ictiofauna de áreas rasas do Complexo Estuarino Baía de Paranaguá, Paraná. Tese de doutorado, Universidade Federal de São Carlos, São Carlos.

FÉLIX, F.C., SPACH, H.L., MORO, P.S., HACKRADT, C.W., QUEIROZ, G.M.L.N. \& HOSTIM-SILVA, M. 2007. Ichthyofauna composition across a wave-energy gradient on southern Brazil beaches. Braz. J. Oceanogr. 55(4):281-292. http://dx.doi.org/10.1590/S167987592007000400005

FIGUEIREDO, J.L., SALLES, A.C.R. \& RABELO, L.B. 2010. Sardinella brasiliensis (Steindachner, 1879) (Teleostei: Clupeidae), nome válido aplicado à sardinha verdadeira no sudeste do Brasil. Pap. Avulsos Zool. 50(18):281-283. http://dx.doi.org/10.1590/S003110492010001800001

FLOETER, S.R., ROCHA, L.A., ROBERTSON, D.R., JOYEUX, J.C., SMITH-VANIZ, W.F., WIRTZ, P., EDWARDS, A.J., BARREIROS, J.P., FERREIRA, C.E.L., GASPARINI, J.L., BRITO, A., FALCÓN, J.M., BOWEN, B.W. \& BERNARDI, G. 2008. Atlantic reef fish biogeography and evolution. J. Biogeogr. 35:22-47. http://dx.doi.org/10.1111/j.13652699.2007.01790.x

FROESE, R. \& PAULY, D. 2010. FishBase. World Wide Web electronic publication. version (07/2010). http://www.fishbase.org/home.htm (último acesso em 27/10/2010).

GIARRIZZO, T. \& KRUMME, U. 2007. Spatial differences and seasonal cyclicity in the intertidal fish fauna from four mangrove creeks in a salinity zone of the Curuçá estuary, north Brazil. B. Mar. Sci. 80(3):739-754.

GODEFROID, R.S., HOFSTAETTER, M. \& SPACH, H.L. 1997. Structure of the fish assemblage in the surf zone of the beach at Pontal do Sul, Paraná. Nerítica 11:77-93.

GODEFROID, R.S., HOFSTAETTER, M. \& SPACH, H.L. 1999. Larval fish in the surf zone of Pontal do Sul beach, Pontal do Paraná, Brazil. Rev. Bras. Zool. 16(4):1005-1011. http://dx.doi.org/10.1590/S010181751999000400010

GRAY, G.A. \& WINN, H.E. 1961. Reproductive ecology and sound production of scarecrow toadfish, Opsanus tau. Ecology 42(2):274-282.

HACKRADT, C.W., PICHLER, H.A., FÉLIX, F., SCHWARZ JUNIOR, R., SILVA, L.O. \& SPACH, H.L. 2009. A estrutura da comunidade de peixes em praias de baixa energia do Complexo Estuarino da Baía de Paranaguá, Brasil. Rev. Bras. Zoocienc. 11(3):231-242.

HERCOS, A.P. 2006. Diversidade e variabilidade espaço-temporal da ictiofauna da região estuarina do rio Curuçá município de Curuçá, Pará Brasil. Dissertação de mestrado, Universidade Federal do Pará, Belém.

IGNÁCIO, J.M. \& SPACH, H.L. 2009. Variação entre o dia e a noite nas características da ictiofauna da infralitoral raso do Maciel, Baía de Paranaguá, Paraná. Rev. Bras. Zoocienc. 11(1):25-37.

INTERNATIONAL UNION FOR CONSERVATION OF NATURE - IUCN. 2012. IUCN Red List of Threatened Species. http:// www.iucnredlist.org/ (ultimo acesso em 28/02/2012).

JAUREGUIZAR, A.J., MENNI, R., GUERRERO, R. \& LASTA, C. 2004. Environmental factors structuring fish communities of the Río de la Plata estuary. Fish. Res. 66:195-211. http://dx.doi.org/10.1016/S01657836(03)00200-5

KNOPPERS, B.A., BRANDINI, F.P. \& THAMM, C.A. 1987. Ecological studies in the Bay of Paranaguá. II.Some physical and chemical characteristics. Nerítica 2(1):1-36.

LANA, P.C., MARONE, E., LOPES, R.M. \& MACHADO, E.C. 2001. The Subtropical Estuarine Complex of Paranaguá Bay, Brazil. In Coastal Marine Ecosystems of Latin America (U. Seeliger \& B. Kjerfve, eds). Springer-Verlag, Berlin, p.131-145.
LUIZ JUNIOR, O.J., CARVALHO-FILHO, A., FERREIRA, C.E.L., FLOETER, S.R., GASPARINI, J.L. \& SAZIMA, I. 2008. The reef fish assemblage of the Laje de Santos Marine State Park, Southwestern Atlantic:annotated checklist with comments on abundance, distribution, trophic structure, symbiotic associations, and conservation. Zootaxa 1807:1-25.

MAGURRAN, A.E. 2003. Measuring Biological Diversity. Blackwell Publishing, Oxford.

MARCENIUK, A.P. 2005. Chave para identificação das espécies de bagres marinhos (Siluriformes, Ariidae) da costa brasileira. B. Inst. Pesca 31(2):89-101.

MARONE, E., MACHADO, E.C., LOPES, R.M. \& SILVA, E.T. 2005. Landocean fluxes in the Paranaguá Bay estuarine system, Southern Brazil. Braz. J. Oceanogr. 53:169-181.

MENEZES, N.A., BUCKUP, P.A., FIGUEIREDO, J.L. \& MOURA, R.L. 2003. Catálogo de espécies de peixes marinhos do Brasil. Museu de Zoologia da Universidade de São Paulo, São Paulo.

MENEZES, N.A., OLIVEIRA, C. \& NIRCHIO, M. 2010. An old taxonomic dilemma: the identity of the western south Atlantic lebranche mullet (Teleostei: Perciformes: Mugilidae). Zootaxa 2519:59-68.

NAKAYAMA, P. 2004. Variação temporal e espacial da composição da ictiofauna demersal do infralitoral raso da Baía de Paranaguá, PR, Brasil. Dissertação de mestrado, Universidade Federal de São Carlos, São Carlos.

NOERNBERG, M.A., LAUTERT, L.F.C., ARAÚJO, A.D., MARONE, E., ANGELOTTI, R., NETTO JUNIOR, J.P.B. \& KRUG, L.A. 2004. Remote Sensing and GIS Integration for Modelling the Paranaguá Estuarine Complex - Brazil. J. Coast. Res. 39 (SI):1627-1631.

OLIVEIRA NETO, J.F, SPACH, H.L., SCHWARZ JUNIOR, R. \& PICHLER, H.A. 2008. Diel variation in fish assemblages in tidal creeks in southern Brazil. Braz. J. Biol. 68(1):37-43. http://dx.doi.org/10.1590/S151969842008000100006

PICHLER, H.A., GODEFROID, R.S., MOTTA, R.O., SPACH, H.L., MAGGI, A.S. \& PASSOS, A.C. 2008. Influência do artefato de pesca na caracterização da ictiofauna em planícies de maré. Cad. Esc. Saúde - Cienc. Biol. Unibrasil 1:1-27.

PICHLER, H.A. 2009. Utilização de planícies de maré pela ictiofauna em dois setores do estuário de Paranaguá, sul do Brasil. Tese de doutorado, Universidade Federal do Paraná, Curitiba.

PINHEIRO, P.C. 1999. Dinâmica das comunidades de peixes em três áreas amostrais da Ilha do Mel, Baía de Paranaguá, Paraná, Brasil. Dissertação de mestrado, Universidade Federal do Paraná, Curitiba, Paraná.

QUEIROZ, G.M.L.N., SPACH, H.L., SOBOLEWSKI-MORELOS, M. \& SCHWARZ JUNIOR, R. 2007. A ictiofauna demersal de áreas com diferentes níveis de ocupação humana, no estuário de Paranaguá. Arq. Cienc. Mar. 40(2):80-91.

REIS-FILHO, J.A., NUNES, J.A.C.C. \& FERREIRA, A. 2010. Estuarine ichthyofauna of the Paraguaçu River, Todos os Santos Bay, Bahia, Brazil. Biota Neotrop. 10(4): http://www.biotaneotropica.org.br/v10n4/pt/abstra ct?inventory+bn03610042010 (último acesso em 06/09/2011).

SANTOS, C., SCHWARZ JUNIOR, R., OLIVEIRA NETO, J.F. \& SPACH, H.L. 2002. A ictiofauna em duas planícies de maré do setor euhalino da Baía de Paranaguá, PR. Bol. Inst. Pesca 28(1):49-60.

SÃO PAULO. Secretaria do Meio Ambiente do Estado de São Paulo - SEMA/ SP. 2010. Decreto ${ }^{\circ}{ }^{5} 56.031$, de 20 de julho de 2010. Declara as Espécies da Fauna Silvestre Ameaçadas, as Quase Ameaçadas, as Colapsadas, Sobrexplotadas, Ameaçadas de Sobrexplotação e com dados insuficientes para avaliação no Estado de São Paulo e dá providências correlatas. Diário Oficial do Estado de São Paulo, São Paulo, Poder Executivo, 21 jul. 2010. Seção 1. http://www.cetesb.sp.gov.br/licenciamentoo/legislacao/estadual/ decretos/2010_Dec_Est_56031.pdf. (último acesso em 16/02/2011).

SARPEDONTI, V., ANUNCIAÇÃO, E.M.S. \& NAHUM, V.J.I. 2008. Ichthyoplankton variation in two mangrove creeks of the Curuçá estuary, Pará, Brazil. Ecotrópicos 21(1):1-12. 
SCHWARZ JUNIOR, R., FRANCO, A.C.N.P., SPACH, H.L., SANTOS, C., PICHLER, H.A. \& QUEIROZ, G.M.L.N. 2007. Variação da estrutura especial da ictiofauna demersal capturada com rede de arrasto de porta na Baía dos Pinheiros, PR. Bol. Inst. Pesca 33(2):157-169.

SHEAVES, M. 2006. Scale-dependent variation in composition of fish fauna among sandy tropical estuarine embayments. Mar. Ecol. Prog. Ser. 310:173-184. http://dx.doi.org/10.3354/meps310173

SMITH, W.L. \& CRAIG, M.T. 2007. Casting the Percomorph net widely:the importance of broad taxonomic sampling in the search for the placement of the serranid and percid fishes. Copeia 2007(1):35-55. http://dx.doi. org/10.1643/0045-8511(2007)7[35:CTPNWT]2.0.CO;2

SPACH, H.L., SANTOS, C. \& GODEFROID, R.S. 2003. Padrões temporais na assembléia de peixes na gamboa do Sucuriú, Baía de Paranaguá, Brasil. Rev. Bras. Zool. 20(4):591-600. http://dx.doi.org/10.1590/S010181752003000400005

SPACH, H.L., FÉLIX, F.C., HACKRADT, C.W., LAUFER, D.C., MORO, P.S. \& CATTANII, A.P. 2006. Utilização de ambientes rasos por peixes na Baía de Antonina, Paraná. Biociências 14(2):125-135.

SPACH, H.L., GODEFROID, R.S., SANTOS, C., SCHWARZ JUNIOR, R. \& QUEIROZ, G.M.L.N. 2004a. Temporal variation in fish assemblage composition on a tidal flat. Braz. J. Oceanogr. 52(1):47-58.

SPACH, H.L., SANTOS, C., GODEFROID, R.S., NARDI, M. \& CUNHA, F. 2004b. A study of the fish community structure in a tidal creek. Braz. J. Biol. 64(2):337-351.
SPACH, H.L., SANTOS, C., PICHLER, H.A., IGNÁCIO, J.M., STOIEV, S.B. \& BERNARDO, C. 2007. Padrões estruturais da assembléia de peixes em duas áreas do Canal da Cotinga, Baía de Paranaguá, Paraná, Brasil. Bioikos 21(2):57-67.

STEFANONI, M.F. 2007. Inter-relações tróficas e ictiofauna de uma praia da Ilha das Peças, Paraná. Dissertação de mestrado, Universidade Federal do Paraná, Curitiba.

VENDEL, A.L., SPACH, H.L., LOPES, S.G. \& SANTOS, C. 2002. Structure and Dynamics of fish assemblage in a tidal creek environment. Braz. Arch. Biol. Technol. 45(3):365-373. http://dx.doi.org/10.1590/S151689132002000300015

VENDEL, A.L., LOPES, S.G., SANTOS, C. \& SPACH, H.L. 2003. Fish Assemblages in a tidal flat. Braz. Arch. Biol. Technol. 46:233-242. http:// dx.doi.org/10.1590/S1516-89132003000200015

VIEIRA, J.P. \& MUSICK, J.A. 1994. Fish faunal composition in warmtemperate and tropical estuaries of western Atlantic. Atlântica 16:31-53.

VILAR, C.C., SPACH, H.L. \& SANTOS, L.O. 2011. Fish fauna of Baía da Babitonga (southern Brazil), with remarks on species abundance, ontogenic stage and conservation status. Zootaxa 2734:40-52.

VITULE, J.R.S., UMBRIA, S.C. \& ARANHA, J.M.R. 2006. Introduction of the African catfish Clarias gariepinus (BURCHELL, 1822) into Southern Brazil. Biol. Invasions 8:677-681. http://dx.doi.org/10.1007/ s10530-005-2535-8 